Recherches en didactique des langues et des cultures

Les cahiers de l'Acedle

11-2| 2014

Plurilinguisme(s) et entreprise : enjeux didactiques

\title{
Pour une meilleure évaluation de la compétence lexicale dans les écrits créatifs en FLE
}

Christèle Maizonniaux

\section{(2) OpenEdition}

Journals

Édition électronique

URL : http://journals.openedition.org/rdlc/1810

DOI : $10.4000 /$ rdlc. 1810

ISSN : 1958-5772

Éditeur

ACEDLE

Référence électronique

Christèle Maizonniaux, «Pour une meilleure évaluation de la compétence lexicale dans les écrits créatifs en FLE », Recherches en didactique des lanques et des cultures [En ligne], 11-2 | 2014, mis en ligne le 07 juin 2014, consulté le 01 mai 2019. URL : http://journals.openedition.org/rdlc/1810 ; DOI : $10.4000 /$ rdlc. 1810

Ce document a été généré automatiquement le 1 mai 2019.

\section{$\oplus \Theta \Theta \Theta$}

Recherches en didactique des langues et des cultures is licensed under a Creative Commons AttributionNonCommercial-NoDerivatives 4.0 International License 


\title{
Pour une meilleure évaluation de la compétence lexicale dans les écrits créatifs en FLE
}

\author{
Christèle Maizonniaux
}

\section{Introduction}

1 Le domaine de recherche auquel nous nous intéresserons ici est celui de l'écriture créative en langue étrangère et plus particulièrement de l'évaluation de la compétence lexicale dans des exercices d'écriture sous contrainte, d'appropriation et de détournements des textes. Dans le cadre d'un projet de recherche doctorale, qui a débuté en 2009 à l'Australian National University (ANU), des ouvrages de littérature d'enfance et de jeunesse ont été proposés à des apprenants de Français Langue Étrangère (FLE) à l'université au niveau ab initio. Pour les apprenants participant à la recherche, on estime en général qu'ils atteignent le niveau $\mathrm{A} 2$ au cours ou à la fin du premier semestre de deuxième année. C'est au cours de ce semestre qu'a été conduite la présente recherche ${ }^{1}$. La première partie du corpus proposé aux apprenants était composée d'albums et de récits illustrés, réécritures d'un conte connu de tous, Le Petit Chaperon Rouge ainsi que de documents annexes éclairant des aspects culturels tels que l'origine du conte traditionnel ou les représentations du loup en France et dans la littérature. Notre approche s'inscrivant dans le cadre de la lecture-écriture, nous avons proposé aux apprenants de réécrire leur propre version du conte après la lecture des iconotextes et documents proposés. L'examen de ces réécritures doit permettre d'observer quels transferts ont eu lieu au niveau du lexique et comment ces transferts s'inscrivent dans l'écriture. Il doit également mettre en lumière la part des apports personnels de l'apprenant. Nous préciserons ici que l'étude fait partie intégrante d'une recherche plus vaste dont l'objectif est de montrer la pertinence de l'utilisation de supports multimodaux pour la jeunesse pour l'enseignement du français langue étrangère à ce niveau d'apprentissage ainsi que la capacité de tâches d'écriture créative à susciter l'engagement d'apprenants et la 
production de textes à la fois riches et pertinents témoignant d'une lecture attentive, personnelle et singulière des iconotextes choisis.

\section{Contexte théorique}

2 Nous situons notre recherche dans le cadre de la lecture-écriture en langue étrangère. En FLE comme en anglais langue étrangère, des activités ont été proposées qui invitent les apprenants à effectuer diverses tâches d'écriture créative à partir de lectures littéraires (voir les travaux de Collie \& Slater, 1987; Albert \& Souchon 2000). Encore appelés exercices d'écriture sous contraintes, ces tâches consistent par exemple en des greffes, des réécritures qui peuvent être des parodies ou encore des pastiches. Si la recherche en français langue maternelle s'intéresse particulièrement ces dernières années à l'apport de ces activités d'écriture (voir par exemple l'ouvrage coordonné par J. L. Dufays et Sylvie Plane L'écriture de fiction en classe de français (Dufays \& Plane, 2009)), peu d'études ont, en revanche, à ce jour été effectuées dans le champ de l'enseignement-apprentissage des langues étrangères. Certains didacticiens du FLE soulignent le fait « qu'il est encore trop rare dans les méthodes qu'on fasse écrire les apprenants dans le but de développer une véritable compétence textuelle " (Cuq \& Gruca, 2005: 184). Ces mêmes chercheurs soulignent la nécessité de «dresser des passerelles entre la production en langue maternelle et en langue étrangère et de s'inspirer des diverses théories, notamment typologiques, pour instaurer une didactique de l'écrit qui dépasse le stade de la phrase ou du paragraphe.» (Cuq \& Gruca 2005: 184). Le développement de la compétence à raconter, sollicitée en particulier à travers la production de récits nous parait essentielle. Stéphanie Clerc, dans l'ouvrage coordonné par Francine Cicurel et Daniel Véronique, Discours, action et appropriation des langues, souligne l'importance dans la vie de tous les jours de l'aptitude à raconter (Clerc, 2002). Nous posons que des exercices de type réécriture de conte, dans la mesure où ils requièrent de la part des apprenants des savoir et des savoir-faire pour raconter, mettre en forme un récit, consolident l'aptitude à raconter. Par ailleurs, la tâche de réécriture "crée un besoin de mots" (Elalouf \& Kéravent, 2004: 186, cité par Sardier \& Grossmann, 2010: 15) qui permet aux apprenants de recourir au lexique étudié, de réinvestir ce qu'ils ont appris, lu et vu.

3 En effet, les activités d'écritures combinées à la lecture transforment l'apprenant en « ouvrier de la langue et du texte » (Cuq \& Gruca, 2005 :188.) En tant qu'« ouvrier » de la langue, l'apprenant mobilise alors ses connaissances lexicales, parmi lesquelles des termes ou expressions rencontrés dans la lecture qui a précédé la phase d'écriture. On peut alors dire avec Courtillon que ce type de pratique qui permet à l'apprenant « de choisir en partie son lexique, c'est-à-dire d'exprimer son intention de communication, seront très favorables à l'acquisition. » (Courtillon, 1989 : 146-153).

4 Sans revenir de manière détaillée sur les techniques d'acquisition du vocabulaire dans les différentes méthodologies d'enseignement-apprentissage des langues, nous pouvons dire que nous ne nous situons pas dans le prolongement des approches communicatives, dans la mesure où celles-ci « ne favorisent pas le vocabulaire au-delà d'un niveau fonctionnel minimal. ( (Treville, 1996 : 92-110 cité par Cuq et Gruca : 2005 : 408). Nous nous situons en revanche dans la tendance actuelle qui ne conçoit pas l'enseignement du lexique en dehors de toute contextualisation et qui prône le rapprochement entre lexique et culture, à la suite des travaux effectués par Robert Galisson (Galisson, 1989). 
5 Nous avons choisi pour notre analyse de recourir au terme d'" appropriation ». Ce terme est, selon la définition proposée par le dictionnaire de didactique du français langue étrangère et seconde, "employé comme hyperonyme par certains didacticiens qui veulent neutraliser la dichotomie acquisition/apprentissage" (Cuq, 2003: 25-26). Elle désigne ainsi " l'ensemble des conduites de l'apprenant, des plus conscientes et volontaires (ce que d'autres appelleraient apprentissage) au moins conscientes (ce que d'autres appelleraient acquisition)" (Cuq, $2003: 25-26)$.

$6 \quad$ Notre objectif est ici de montrer que des productions écrites de type réécriture proposées à la suite d'une séquence basée sur la lecture de ces supports multimodaux (albums et courts récits illustrés) et de supports annexes, témoignent d'une appropriation du vocabulaire qui est remarquable tant du point de vue du nombre d'occurrences qu'on peut y relever que de la variété du lexique utilisé ou du réemploi judicieux de celui-ci. Dès lors, il s'avère nécessaire de développer un outil de saisie précis pour rendre compte des divers aspects qui témoignent, dans l'écriture, d'une appropriation du lexique par l'apprenant.

7 Nous faisons l'hypothèse que les productions effectuées dans le cadre de la démarche d'écriture créative proposée témoignent chez nos apprenants de savoirs et de savoir-faire généralement constatés chez des apprenants plus avancés dans leur apprentissage de la langue. On pourra citer ici comme exemple l'usage approprié des temps du passé ou encore la capacité à user de synonymes, de paraphrases.

\section{Méthodologie}

8 Les auteurs des productions analysées sont tous des étudiants de français langue étrangère, inscrits à l'ANU. Ces étudiants se trouvent à une période charnière de leur apprentissage, puisqu'ils doivent passer de la lecture d'extraits (qu'ils soient littéraires ou non) à la lecture autonome d'œuvres intégrales ou de textes plus longs et plus complexes de littérature générale et ce, en particulier au second semestre de deuxième année. Il est important de préciser ici que les groupes sont hétérogènes, multilingues et multiculturels puisque l'ANU accueille un grand nombre d'étudiants de la zone Asie-Pacifique. Ils appartiennent à des filières diverses telles que droit, relations internationales, musique ou autre. Pour plus de clarté, nous nous basons ici uniquement sur notre premier corpus de travaux écrits, constitué de réécritures individuelles du conte Le Petit Chaperon rouge. Il est à noter cependant, que les conclusions de l'analyse de notre deuxième corpus (un exercice créatif de type greffe, où il était demandé aux apprenants d'insérer un nouvel épisode dans la trame narrative existante) sont mises en perspective avec les résultats présentés ici à la fin de ce travail. Le présent corpus est constitué de 26 travaux, réalisés par un groupe d'étudiants mixte ( 16 filles et 10 garçons). Parmi ces 26 étudiants, 4 ont une langue maternelle autre que l'anglais ( 2 le chinois, 1 le polonais, 1 le japonais). 10 étudiants sur les 26 participants à l'étude sont issus du cursus ANU de première année qui représente 130 heures de français. Ces travaux ont été réalisés en classe dans le cadre de l'évaluation de mi-semestre et ont compté pour $40 \%$ de la note finale. L'évaluation sommative correspondait à une exigence institutionnelle. Il n'était pas possible matériellement d'envisager une production d'écrits créatifs qui soit dissociée de la phase d'évaluation requise par l'institution. Il est à noter que les travaux analysés constituent "un premier jet", que la durée consacrée à l'exercice était de 60 minutes et que l'usage d'un dictionnaire bilingue était autorisé. Diverses tâches d'écriture ont été réalisées en 
amont que ce soit en classe ou à la maison, et elles consistaient pour certaines d'entre elles à réécrire une partie d'un des textes/documents étudiés. En outre, la démarche proposée aux apprenants incluait des phases de rappel oral (recall activities), invitant les apprenants à produire des textes narratifs oraux en langue cible leur permettant de réutiliser le lexique et les structures nouvellement introduites.

Pour la présente réécriture, la consigne a été donnée en anglais afin de s'assurer d'une compréhension immédiate et sans faille de celle-ci par nos apprenants qui, rappelons-le, atteignent habituellement au cours ou en fin de semestre le niveau A2 du CECR. Voici cette consigne:

Write your own version of Le Petit Chaperon Rouge. You can modify the famous fairy tale as did the authors we studied in class.

You will use past tenses.

The wolf, little red riding hood, his mother and his grandmother will be the characters (or some of the characters of the story)

You can change the storyline and decide to make a detective story or another type of story as did Yvan Pommaux for example.

(200 words minimum)

\section{Comment saisir les différents aspects de l'appropriation du lexique?}

11 Nous avons mentionné plus haut la nécessité d'établir un cadre afin de saisir les différents aspects de l'appropriation du lexique.

Le Cadre Européen Commun de Référence pour les langues (CECR) définit la compétence lexicale comme suit : "il s'agit de la connaissance et de la capacité à utiliser le vocabulaire d'une langue qui se compose (i) d'éléments lexicaux (expressions toutes faites, mots isolés) et (ii) d'éléments grammaticaux (articles, pronoms personnels, etc.) et de la capacité à les utiliser" (Conseil de l'Europe, 2001 : 87).

13 Le CECR propose deux échelles pour saisir l'appropriation du lexique: l'une a pour objectif d'évaluer l'étendue du vocabulaire et l'autre la maîtrise du vocabulaire.

Les critères "étendue" et "maîtrise" sont ceux qui sont généralement considérés comme cruciaux par les enseignants dans l'évaluation traditionnelle que ce soit pour des productions en langue maternelle (Bucheton \& Chabanne, 2002-2003 ; Pilorgé, 2010) ou pour des productions en langue étrangère. L'étendue touche à la quantité et à la variété de vocabulaire présent. La maîtrise est le lieu où l'on observe l'attention portée à l'utilisation de la langue (le contrôle); c'est aussi le lieu où sont signalées les défaillances, où sont identifiés les dysfonctionnements. Ionnidou (2008) fait remarquer que le CECR ne retient pas les différents constituants de la compétence lexicale dans son modèle (tels ceux développés par Laufer (1994: 99) ou par Tréville et Duquête (1996: 98)) et qu'il préfère analyser l'enseignement et l'apprentissage du vocabulaire « avec les compétences communicationnelles correspondantes » (Ionnidou : 63).

Comme le rappelle Ionnidou (2008: 60), Laufer (1994: 99) décline la compétence lexicale en six points, allant de la reconnaissance à l'oral et à l'écrit du mot (et de sa prononciation et orthographie correcte) à la connaissance des rapports du sens du mot avec d'autres mots (synonymie, antonymie, hyponymie) et de ses collocations les plus fréquentes. Tréville et Duquette (1996: 98), pour leur part, retiennent cinq composantes : les composantes linguistique, discursive, socioculturelle, référentielle et stratégique.

16 Sans négliger l'importance des critères d'étendue et de maîtrise retenus par le CECR, il nous a cependant semblé nécessaire d'ajouter un troisième critère : nous l'avons appelé 
"associations". Ce critère sert à identifier les potentialités du texte de l'apprenantscripteur. Il représente la manière dont sont établies, par les apprenants, des associations entre les diverses unités lexicales présentes dans le discours. Ce critère doit rendre compte :

- d'éléments comme la créativité, les effets littéraires, des éléments spécifiques à des productions qui relèvent de l'écriture créative, et donc de l'écriture littéraire ;

- des « résidus » de la lecture qui a eu lieu en aval de la phase d'écriture ;

- d'éléments qui reflètent la manière dont les apprenants se sont appropriés des supports multimodaux et en rendent compte dans une production monomodale (ce que nous appellerons monomodal response after reading a range of multimodal stimuli).

Si l'on s'en réfère aux différents constituants de la compétence lexicale (cf. plus haut Laufer, 1994 et Tréville \& Duquette, 1996), on se rend compte que ce critère d'associations met en évidence, entre autres, la composante stratégique. Celle-ci consiste à adopter des stratégies efficaces pour contourner un problème de communication. Le critère d' associations proposé permet également d'identifier le fait qu'un mot ou une expression est utilisé(e) dans un sens autre que celui rencontré dans la lecture des textes-sources et ce, dans le but de créer une image particulière. On peut alors dire que l'apprenant a surmonté la difficulté liée à la " nature multidimensionnelle des mots » (Laufer 1994 : 99) et qu'il a tiré parti de la connaissance qu'il avait des relations que ce mot entretient avec d'autres mots.

Parallèlement au critère d'association que nous proposons, nous trouvons également celui d'élaboration qui démontre, pour reprendre les termes de Nadège Pandraud, que des apprenants "perçus comme faibles ou usant d'un code restreint ont de véritables potentialités narratives, qui ne sont pas directement observables ou qui peuvent être invisibilisées dans l'école" (2009 : 224). L'élaboration peut se manifester dans un texte de plusieurs manières : il peut s'agir de choix lexicaux et d'associations qui concourent à produire une impression ou un effet sur le lecteur (il s'agit là d'une dimension esthétique de la langue), d'éléments qui contribuent à rendre le texte crédible (à faire qu'on «y croit»), ou encore d'éléments qui concourent à la production d'un texte "résistant", au sens où il ne s'épuise pas à la première lecture.

19 Nous aimerions montrer, à partir de l'analyse de quelques-unes des productions de notre corpus, que la réécriture aboutit à des productions hybrides qui, tout en étant imprégnées par les lectures de supports multimodaux faites en classe, contiennent des éléments qui relèvent de l'apport personnel de l'apprenant. Pour rendre compte de l'appropriation du vocabulaire par l'apprenant-scripteur, nous proposons la grille d'analyse suivante :

Tableau 1 - Grille d'analyse : Appropriation du vocabulaire en langue étrangère : réécriture de conte après lecture de supports multimodaux

\begin{tabular}{|l|l|l|}
\hline $\begin{array}{l}\text { Critères/ } \\
\text { Descripteurs }\end{array}$ & Domaines & Éléments identifiables dans les productions d'apprenants \\
\hline & & Développement d'un champ lexical particulier \\
\hline $\begin{array}{l}\text { Etendue } \\
\text { vocabulaire }\end{array}$ & $\begin{array}{l}\text { Quantité/ } \\
\text { variété }\end{array}$ & Présence d'expressions idiomatiques/de locutions figées \\
\hline & & Présence de vocabulaire spécifique à une culture \\
\hline
\end{tabular}




\begin{tabular}{|c|c|c|}
\hline & & Détails qui participent de la « vraisemblabilisation » du récit \\
\hline & & Vocabulaire utilisé de manière correcte et appropriée \\
\hline \multirow[t]{4}{*}{$\begin{array}{l}\text {-Maîtrise } \mathrm{du} \\
\text { vocabulaire }\end{array}$} & Correction & Correction orthographique \\
\hline & & $\begin{array}{l}\text { Présence peu fréquente de mots en langue } 1 \text { ou de structures } \\
\text { calquées sur la langue } 1\end{array}$ \\
\hline & & Associations qui créent une impression/un effet sur le lecteur \\
\hline & & $\begin{array}{l}\text { Tout ce qui peut être rangé dans la catégorie "dimension } \\
\text { esthétique de la langue" : métaphores, assonances, etc. }\end{array}$ \\
\hline \multirow[t]{2}{*}{$\begin{array}{l}\text {-Associations } \\
\text { lexicales }\end{array}$} & Créativité & $\begin{array}{l}\text { Éléments contribuant à faire du récit un récit "crédible ", } \\
\text { éléments contribuant à la "vraisemblabilisation " du récit } \\
\text { (influence de l'initiation à la pratique du conte oral en classe) }\end{array}$ \\
\hline & & $\begin{array}{l}\text { Tout ce qui contribue à créer un texte "résistant" c'est-à-dire } \\
\text { un texte dont le sens ne s'épuise pas à la première lecture. }\end{array}$ \\
\hline
\end{tabular}

\section{Résultats, développement théorique}

\section{Présentation du corpus et de la démarche proposés}

20 Afin de clarifier notre démarche, nous proposons ci-dessous un tableau récapitulatif des documents étudiés ainsi qu'une synthèse de l'approche dont ils ont fait l'objet.

Tableau 2 - Corpus et démarche proposés

\begin{tabular}{|l|l|l|}
\hline $\begin{array}{l}\text {-Document } \\
\text { source (DS) }\end{array}$ & $\begin{array}{l}\text { Titre et caractéristiques essentielles des } \\
\text { supports de notre corpus }\end{array}$ & Démarche pédagogique \\
\hline •DS1 & $\begin{array}{l}\text { Version simplifiée du conte-source Le Petit } \\
\text { Chaperon Rouge avec fin des frères Grimm et } \\
\text { fin de Charles Perrault extrait de la } \\
\text { méthode Tatou le Matou (Hachette). }\end{array}$ & $\begin{array}{l}\text { Texte donné sous forme audio puis } \\
\text { en lecture en classe + recall task } \\
\text { (tâche de rappel à l'oral) }\end{array}$ \\
\hline -DS2 & $\begin{array}{l}\text { Album John Chatterton détective d'Yvan } \\
\text { Pommaux. Réécriture parodique du conte. } \\
\text { Référence au film noir, aux arts plastiques, } \\
\text { au conte. }\end{array}$ & $\begin{array}{l}\text { Lecture/questions en classe. } \\
\text { - Travaux de groupe en classe : } \\
\text { travail ciblé : par exemple un des } \\
\text { aspects des albums à creuser tel } \\
\text { que la relation texte/image, }\end{array}$ \\
\hline
\end{tabular}




\begin{tabular}{|l|l|l|}
\hline •DS3 & $\begin{array}{l}\text { Récit illustré Le Petit Chaperon Vert de Nadja } \\
\text { et Solotareff. Parodie du conte. Récits } \\
\text { enchâssés. }\end{array}$ & $\begin{array}{l}\text { Fiche à remplir à la maison. } \\
\text { Lecture/questions en classe. } \\
\text {-Dâches d'écriture créative } \\
\text { individuelle ou en binôme. }\end{array}$ \\
\hline & $\begin{array}{l}\text { Documents annexes authentiques } \\
\text { concernant l'histoire du conte et/ou } \\
\text { l'histoire du loup (articles de journaux, } \\
\text { images, notice bibliographiques, extraits } \\
\text { d'ouvrages de littérature générale }\end{array}$ & $\begin{array}{l}\text { Documents donnés en lecture } \\
\text { optionnelle à la maison. Exposés en } \\
\text { classe ou travail par petits groupes. }\end{array}$ \\
\hline
\end{tabular}

21 Notre objectif n'est pas ici de relever tous les résidus de la lecture présents dans le texte de l'apprenant-scripteur car cet exercice ne conduirait qu'à faire un bilan des éléments mémorisés sans toutefois rendre compte du travail de tissage à l'œuvre. Il s'agit plutôt ici de montrer que l'utilisation de supports multimodaux dans le cadre d'un enseignement de langue étrangère contribue non seulement à une appropriation de connaissances d'ordre culturel mais aussi à une appropriation linguistique et lexicale remarquable.

Notre analyse des 26 productions d'apprenants a nécessité, en raison de l'hétérogénéité $\mathrm{du}$ corpus obtenu, d'opérer une première classification de celles-ci. Le classement proposé par Pintado (Pintado, 2009) et qui consiste en la classification de réécritures d'auteurs reconnus en fonction de leur degré de proximité par rapport au texte-source a été retenu pour notre analyse. Dans notre cas, il permet de distinguer trois types de productions : celles où la part de l'imaginaire est relativement faible (les productions minimales), celles où la part de l'imaginaire est plus importante mais où les éléments principaux du conte source ont été conservés et sont aisément identifiables (les productions équilibrées) et, enfin, celles où l'imaginaire occupe une grande place et où seuls quelques éléments du conte sont encore perceptibles (les productions maximales).

Nous souhaitons ici présenter les diverses formes que prend l'appropriation du lexique et montrer que des traces de cette appropriation sont observables quels qu'aient été les choix narratifs de l'apprenant-scripteur. Dans un premier temps, nous nous concentrerons sur deux exemples tirés de travaux identifiés comme étant des productions minimales. Dans un second temps, nous nous attarderons sur des exemples correspondant, selon nos critères, à des productions équilibrées ou maximales.

\section{Exemples d'appropriation du lexique dans deux productions minimales}

Notons ici que tous les travaux présentés le sont dans leur forme d'origine et que nous n'avons pas modifié le texte proposé par l'apprenant-scripteur. Nous avons signalé par [sic] les erreurs liés à la correction orthographique ou grammaticale. Lorsque l'écart à la norme était trop important pour permettre une compréhension immédiate, nous avons proposé entre crochets une expression plus appropriée. Ont été transcrites en gras dans le texte de l'apprenant, les expressions qui font part la suite l'objet d'un commentaire dans notre analyse. 


\title{
Exemple 1 (Étudiant A6)
}

\begin{abstract}
Il était une fois une petite fille habitait [sic] dans une petite maison. Tout le fois [sic], elle aimait porter rouge [sic]. Rouge chaussures [sic], un rouge chemisier [sic], des rouge pantalons [sic] et un rouge chapeau[sic]. Elle s'appelait le Petit Chaperon Rouge. Un jour sa mère lui dit "ta mère-grand, elle est malade. Est-ce que tu peux porter ce panier à elle [sic] ?" "D'accord", dit la petite fille. "Dans ce panier, il y a des gâteaux et des medicaments [sic]", dit la mère.
\end{abstract}

Le critère maîtrise permet d'identifier les problèmes de langues élémentaires, comme la place de l'adjectif dans le groupe nominal "rouge chaussures, rouge chemisier..." ou encore le fait que le pronom personnel choisi est inapproprié ("porter ce panier à elle"). Même si la version proposée ici par l'apprenant-scripteur est assez pauvre en termes d'associations, de trouvailles, elle témoigne tout de même d'une appropriation lexicale. On voit que l'apprenant s'est approprié le vocabulaire du conte "il était une fois...ta mère-grand...porter ce panier...". On note également qu'il a essayé de retrouver des expressions revues en classe lors de l'étude du document source 2 (vocabulaire des vêtements). Dans cette réécriture, l'apprenant s'est inspiré de la structure du conte-source et a conservé l'alternance récit dialogue.

\section{Exemple 2 (Étudiant A13)}

Nous avons, au début de cet article, précisé que notre groupe est multilingue et hétérogène mais que l'ensemble des apprenants atteint le niveau A2 du CECR au cours ou à la fin du semestre d'étude qui nous concerne. L'étudiant A13, comme le montre l'extrait ci-dessous, fait partie de ces apprenants plus avancés dans la langue cible. Il a tout comme l'étudiant A6, opté pour une production minimale.

\begin{abstract}
Une fois [sic], il y avait une petite fille que tout le monde appelait "le Petit Chaperon rouge" car elle avait une sorte de couvre-chef qu'elle portait toujours. Un jour sa mère lui demandait [sic] d'apporter un panier de nourriture et médicaments à sa grand-mère qui était très malade. Alors elle partit après avoir mis son chaperon rouge. Dans la forêt, elle rencontra un mauvais loup noir. Elle se dit "le loup est très méchant! Il a mangé beaucoup d'enfants et leurs grands-parents! Je dois le punir !"
\end{abstract}

27 A l'inverse de la précédente cette production minimale est caractérisée par un niveau de maîtrise élevé (peu d'erreurs orthographiques, seulement quelques erreurs de temps). Le texte produit est très proche, du moins au début, de l'hypotexte ou texte d'appui, Le Petit Chaperon Vert (DS3), même si l'apprenant a fait le choix de ne pas produire un récit enchâssé. Cette production peut être considérée comme étant celle d'un apprenant qui fait montre d'une certaine prudence, mais elle témoigne également de transferts nombreux, d'une précision et d'une attention dans la lecture et sans aucun doute de la mémorisation par cœur de phrases entières. Par ailleurs, il convient de noter ici l'habileté dont fait preuve l'apprenant dans le réemploi des structures et du lexique rencontrés. Citons par exemple "elle partit après avoir mis son chaperon rouge" (DS3: "elle partit courageusement après avoir mis son chaperon vert"). Citons encore "elle avait une sorte de couvre-chef" (qui reprend à la fois DS3 : "elle portait une sorte de capuchon pointu et vert" et l'expression "couvre-chef" rencontrée lors d'un travail sur le lexique fait en classe qui portait sur des synonymes du mot "capuchon"). Ici l'emploi de "couvre-chef" témoigne néanmoins d'un problème de conscience du niveau de langue approprié. 

langue. Les erreurs sont nombreuses au point qu'elles constituent une entrave à la compréhension. Un correcteur ayant établi des critères basés sur la correction de la langue aurait tendance ici à se focaliser sur les erreurs, nombreuses, et à sanctionner l'apprenant. Néanmoins une analyse plus précise du texte révèle une appropriation du point de vue lexical ainsi que des potentialités.

Cette dernière observation témoignant d'un répertoire assez large chez l'étudiant A13 nous amène à considérer l'étendue lexicale, ou bien le critère de l'étendue. Notons par exemple l'effort fait par cet apprenant pour ne pas se répéter (un mauvais loup noir/loup très méchant) et ce, malgré la relative brièveté de la production qui ne compte que 226 mots alors que la longueur moyenne des productions du corpus analysé est de 308 mots. Notons également que ce scripteur a intégré les recommandations stylistiques incitant les apprenants à éviter en français les répétitions, ce qui, dans d'autres langues, ne constitue pas nécessairement un défaut majeur.

Chez ce scripteur dont nous savons qu'il est de langue maternelle chinoise, les réemplois nombreux, l'utilisation appropriée des pronoms personnels, la précision grammaticale sont sans doute la marque de sa volonté de bien faire. En outre, il est possible que le scrupuleux travail de mémorisation constaté ou encore le respect de la consigne soient à rapporter aux cultures éducatives du milieu/pays d'origine de cet apprenant dont nous savons qu'elles accordent une importance capitale à ces deux aspects (Bouvier, 2003).

\section{Exemples d'appropriation du lexique dans des productions équilibrées ou maximales}

\section{Exemple 3 (Étudiant A3)}

Dans la version présentée ci-dessous, l'apprenant-scripteur a fait le choix de modifier la perspective narrative. C'est le loup qui parle. Voyons maintenant quelles sont les traces de l'appropriation lexicale dans un extrait de ce texte :

\begin{abstract}
Titre: C'est ça la vie d'un loup
Il était une fois je courrait [sic] par le forêt [sic] à partir d'un chasseur [sic] [un jour je courrais à travers la forêt, voulant échapper à un chasseur]. La nuit est sur le point de tomber. Quelque chose rouge ai attire [sic] ma attention [sic] [quelque chose de rouge a attiré mon attention]. C'était une jeune fille qui portant [sic] un chapeau rouge et pointu. Elle cueillait les fleurs et les girolles et courrait [sic] après les papillons. Elle ma [sic] délectera. [Je vais m'en délecter.] J'ai très afflamé [sic] parce que c'était trois jours [sic] que j'avais pas mangé [sic] [je suis/j'étais ( ?) très affamé parce que cela fait (faisait) trois jours que je n'ai pas mangé]. J'ai bien envie de lui [sic] mangerai [sic] [la manger] immédiatement mais je n'osa [sic] a cause de ses boucherons[sic] [bucherons] qui était[sic] dans le bois. Aussitôt Plutôt [sic] j'ai arrêté lui parler, nonchalentement [sic], et développe [sic] un projet astucieux.
\end{abstract}

En effet, on ne peut qualifier le lexique ici de "pauvre". On constate ainsi de nombreux transferts à partir de DS2 ou DS3, par exemple: "quelque chose rouge ai attire [sic] ma attention [sic]". Comme le montre la reproduction de l'image ci-dessous, cette phrase fait référence à une image d'un des albums étudiés (John Chatterton détective d'Yvan Pommaux) et reprend le lexique vu en classe. 
Figure 1 - John Chatterton détective, pp. 12-13.

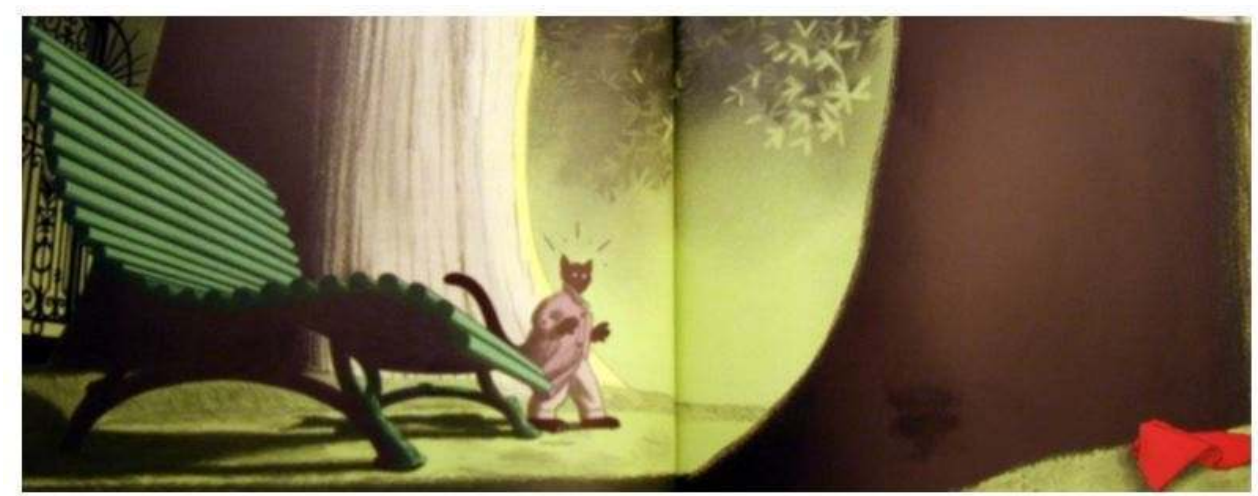
plusieurs phrases du texte de l'apprenante font, elles, écho à DS3 (Le Petit Chaperon Vert de Solotareff et Nadja). Citons par exemple : "c'était une jeune fille qui portant [sic] un chapeau rouge et pointu" qui rappelle "il était une fois une petite fille que tout le monde appelait le petit chaperon vert parce qu'elle portait une sorte de capuchon pointu et vert" ou encore "elle cueillait les fleurs et les girolles et courrait après les papillons" qui reprend "cueillait des fleurs et ramassait des girolles" (DS3) et "courrait après les papillons" (DS4). observe des détails qui contribuent à la «vraisemblabilisation » du texte et qui ont une efficacité esthétique sur le lecteur : "la nuit est sur le point de tomber", "c'était une jeune fille qui portant un chapeau rouge et pointu". exprimer les pensées du loup narrateur de cette version: "elle m'a délectera [sic]". L'intégration du style indirect libre dans la narration relève ici d'une véritable compétence et il n'est pas à exclure qu'elle provienne d'un savoir-faire antérieurement présent en langue maternelle ou encore acquis dans son parcours scolaire ou universitaire.

Enfin, on observe une mise en tension à la fin du texte: le paragraphe se termine par " plutôt j'ai arrêté lui parler, nonchalentement [sic] et développe [sic] un projet astucieux".

Il est donc crucial dans le cas de la présente production de souligner l'inadéquation et l'insuffisance des critères traditionnels (maîtrise/étendue) pour rendre compte des potentialités du texte. Dans le cas présent, ces critères permettent surtout d'identifier les manques ou de mettre en avant une situation paradoxale, où un texte peut s'avérer pertinent tout en présentant de graves lacunes au niveau de la correction de la langue. Par conséquent, on peut dire ici que les difficultés rencontrées pour l'évaluation de cette production écrite de type créatif rejoignent d'une certaine manière, en langue 2, les difficultés rencontrées en langue 1 pour l'évaluation de l'écriture littéraire. ${ }^{2}$ Il convient toutefois de noter qu'au niveau universitaire le fait de parvenir à exprimer ses idées en langue étrangère, même avec des erreurs de langue ou de syntaxe, mérite d'être reconnu et valorisé et on doit, par conséquent, tenir compte de cet élément lorsque sont définis les critères d'évaluation. 


\section{Exemple 4 (Étudiant A21)} tout d'abord à la transposition de l'histoire dans l'univers citadin, pensons ensuite à l'idée que le garçon dont il question ici porte un t-shirt à l'effigie du loup à l'instar du loup de Pommaux qui arbore sur l'image une bague à sa propre effigie. Par conséquent, si le garçon agresseur porte une chemise à l'effigie du loup, l'idée est que c'est lui le loup.

Figure 2 - John Chatterton détective, pp. 20-21.
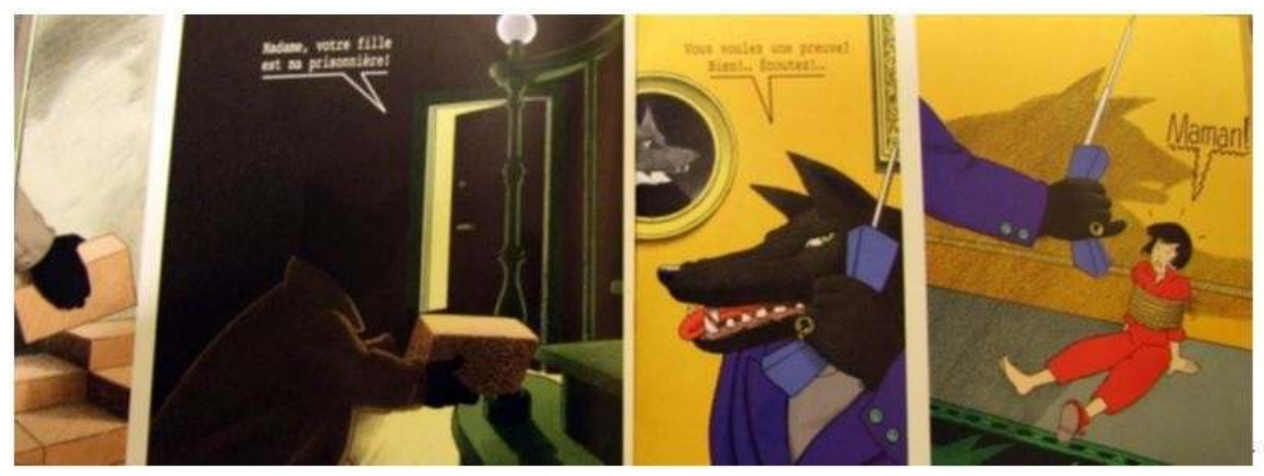

Autre élément remarquable dans le texte de l'étudiant A21, c'est la force du récit, le rendu de l'agression:

"Hé, arrete [sic] !" a hurlé Pierre. Le garçon s'est retourné. Pierre a vu qu'il portait un chemise [sic] avec une picture [sic] d'un loup. Tout à coup, le garçon a empoigné le chapeau rouge de Pierre et s'est enfui! Pierre a commencé à poursuivre le garçon mais il était trop rapide. Pierre s'est promené tristement à la maison de sa grandmère [sic].

41 Comme le montre cet exemple, ce sont donc bien ici les associations qui donnent sa force au récit, qui créent une dynamique. Du point de vue textuel, on observe la présence d'un narrateur omniscient, on observe également l'alternance entre récit et discours direct. Les détails contribuent à rendre le récit vraisemblable. Et, bien sûr on ne peut pas lire le texte de l'apprenant-scripteur sans noter la référence au conte musical "Pierre et le loup" de Prokofiev, qui, précisons-le, n'a pas été étudié en classe. Le prénom comme le note Catherine Tauveron (Tauveron, 1995), constitue une propriété fondamentale des personnages. La qualité du récit tient également à un bon niveau de maîtrise en dehors de quelques incorrections et aussi à un bon degré d'étendue. 


\title{
Exemple 5 (Étudiant A15)
} d'un texte autour d'un champ lexical permet de conférer à une production une force indéniable. On observe dans l'extrait présenté ci-dessous comment est développé par cet apprenant l'idée d'un chaperon criminel. Il y a dans ce texte parfaite concordance entre le nom du personnage (la symbolique du noir), son allure et ce qu'il est. Le lexique autour du thème criminelest développé puisqu'on trouve des termes comme "meurtrière des loups », « détruire tous les loups au monde », « empoisonner ", " poignarder ». Les termes « ennemi », « mitrailleuse » ou « tuer » y font écho plus loin. Bien entendu, l'inférence de savoir-faire déjà acquis dans la langue 1 et le transfert de compétences littéraires de la langue 1 dans la langue 2 ne sont pas à exclure ici. Nous reviendrons dans la suite de cette analyse sur cette question.

\begin{abstract}
Il était une fois quand une petite fille vivait [sic] qui s'appelle "Le Petit Chaperon Noir". Elle s'habillait à tout le noir [tout en noir], $\boldsymbol{y}$ compris un chaperon noir. Le Petit Chaperon noir était une meurtrière des loups[sic]. Sa raison d'être était détruire tous les loups au monde. Elle les empoisonnait, elle les poignardait et elle les étranglait [...] Le Petit Chaperon noir se pensait [sic], "Hmmm... la maison de ma grand-mère est dans la forêt. Cette forêt-là est le foyer de mon ennemi - le loup noir! [...] "Ah disait [sic] le loup. "Mon dîner arrive!" "Pas aujourd'hui disait [sic] le Petit Chaperon Noir, et de leur panier elles ont pris des mitrailleuses! Elles ont tué le loup et elles ont vécu heureusement [heureuses] après ça.
\end{abstract}

\section{Exemple 6 (Étudiant A5)}

Notre dernier exemple est celui de l'étudiant A5, une apprenante qui a fait le choix d'une transposition du conte dans l'époque contemporaine. A l'instar de plusieurs autres exemples déjà présentés, cette réécriture est marquée par des emprunts aux iconotextes étudiés en classe. Cette fois, il s'agit de l'emprunt de motifs, à savoir ceux du mensonge et de la ruse présents notamment dans DS3. Les emprunts à cet ouvrage ne s'arrêtent pas là, puisque le personnage principal est, comme celui de Solotareff et Nadja, une fille rusée. Cela dit et, de manière tout à fait intéressante et originale, l'apprenante ajoute d'autres traits particuliers et singuliers à son personnage qui font d'elle une héroïne semblable à celles de romans modernes pour adultes.

\begin{abstract}
Il y avait une fois une fille qui s'appellait [sic] le Petit Chaperon Rouge. Tout le monde pensait qu'elle était naïve et douce en particulier sa mère, mais le Petit Chaperon Rouge n'était pas ainsi. Elle avait beaucoup de petits amis au même temps [sic] et elle fumait sous les ponts tous les jours. Mais comme elle aimait son image d'innocence [sic], elle faisait tout que [sic] sa mère lui demandait. Un jour, sa mère lui dit [...] Plus tard, le petit Chaperon Rouge créa une histoire avec sa grand-mère qui se conformait à son image - et elle vivait [sic] encore une vie très agréable avec sa mère.
\end{abstract}

Dans la partie centrale du texte (non reproduite ici), on trouve de nombreux transferts d'expressions rencontrées dans les documents sources, comme par exemple : "vous allez par ce chemin-là, et j'irai par ce chemin ci et nous verrons qui arrivera le premier". Ce texte qui, à l'instar de celui de Solotareff, mêle des éléments de langage vieillis et d'autres plus contemporains, a un caractère hybride. Si ce procédé d'écriture a été souligné lors de la 
lecture collective de textes en classe, nous ne savons pas si l'apprenante en use, pour sa part, de manière consciente.

En outre, l'élaboration autour du personnage du chaperon rouge, le travail autour de la dichotomie entre apparences ("naïve et douce") et réalité ("mais le petit Chaperon Rouge n'était pas ainsi"; "elle avait beaucoup de petits amis... elle fumait sous les ponts") est particulièrement intéressante ici et bien développée. On pense à un transfert de la compétence littéraire de la langue 1 à la langue 2, même si, à ce jour, peu de recherches ont été effectuées dans ce domaine. Cependant un certain nombre de recherches (Odlin, 1989 ; Lefrançois, 2001) mettent en évidence des effets de transfert, et ce aussi bien dans les processus de bas niveau que de haut niveau et concluent à un transfert des schémas culturels propres à la littératie ou à la rhétorique de la langue 1 (Lefrançois, 2001 ; Odlin 1989 : 134-136).

Pour ces productions équilibrées ou maximales, on peut sans doute constater une part plus importante de l'imaginaire, des apports personnels de l'apprenant et sans doute, une tendance à une plus grande variété de lexique due au fait que l'apprenant-scripteur convoque d'autres thèmes et champs lexicaux et que, dans la majeure partie des cas, il a une bonne maîtrise de la langue.

\section{Conclusion}

Pour conclure, on peut dire que la tâche proposée de type « écriture sous contrainte » (ici une réécriture de conte), a suscité le besoin, « la motivation » au sens où l'entend Corson (1985 cité par Nation, 2001 : 183) de réutiliser le vocabulaire rencontré.

On constate une appropriation remarquable des structures lues, des images vues, du lexique introduit, et ce, quels que soient les choix effectués par l'apprenant-scripteur. Ceci témoigne, de notre point de vue, d'une implication dans la lecture des ouvrages retenus et dans la démarche proposée. En outre, dans les productions qui s'éloignent du conte-source, on observe la présence de techniques utilisées par les auteurs de réécritures postmodernes telles que jeux avec l'hypotexte ou emprunts d'éléments de langage vieillis.

Il ne fait nul doute que les activités orales incluses dans la démarche, et en particulier celles de rappel où les apprenants ont été invités à remobiliser du vocabulaire ainsi qu'à relier habilement leur propre contribution à celle d'autres interlocuteurs, ont été importantes dans le processus d'appropriation et de réemploi du vocabulaire dans un premier temps. En outre, l'introduction que nous avons proposée à quelques-unes des techniques du conteur, a sans doute joué un rôle important dans la manière d'exprimer les idées et favorisé le réemploi, en situation de production, du lexique rencontré lors des lectures.

50 La diversité des productions (tant en termes de longueur, de choix énonciatif, de proximité par rapport à un ou des documents-source ou au conte-source) permet de conclure que même dans le cas où une copie présente des faiblesses sur le plan linguistique, il y a des indicateurs d'appropriation du lexique. Ces éléments permettent de conclure à un engagement de l'apprenant-scripteur quel que soit son niveau linguistique, ses choix narratifs et les qualités littéraires de sa production.

51 Pour ce qui est de la compétence lexicale, nos apprenants démontrent donc un certain nombre d'habiletés, qui, si l'on s'en réfère aux grilles de compétence du CECR, sont celles 
d'un niveau plus élevé que celui que l'on estime généralement être le leur (Rappelons ici qu'on estime, en général, que les étudiants de deuxième année de français à l'ANU atteignent le niveau $\mathrm{A} 2^{3}$ au cours ou à la fin du premier semestre de deuxième année). On peut donc considérer à la lecture de la grille concernant l'écriture créative (Conseil de l'Europe, 2001:52) que le niveau des apprenants du groupe ayant une bonne maîtrise de la langue correspond davantage au niveau C1 (tâche ambitieuse, remplie de manière satisfaisante).

Pour de futures recherches, il serait pertinent, compte tenu du fait que notre démarche n'envisage pas de travail de reprise de ces productions par leurs auteurs, d'analyser ce qu'un travail basé sur l'autocorrection ou d'autres techniques visant à améliorer les textes au niveau de la maîtrise, de l'étendue mais également de ce que nous avons appelé "les associations" pourrait apporter. Il serait également pertinent de soumettre les écrits produits à des lecteurs « natifs » non enseignants pour observer les réactions de ceux-ci. Travailler à la réécriture des textes produits permettrait aux apprenants d'inscrire leur travail dans la durée et les inviterait à poursuivre et intensifier le travail entamé.

\section{BIBLIOGRAPHIE}

Albert, M.-C. \& Souchon, M. (2000). Les textes littéraires en classe de langue. Paris : Hachette Livre. Bucheton, D. \& Chabanne, J.-C. (2002-2003). "Un autre regard sur les écrits des élèves : évaluer autrement." Repères $n^{\circ} 3, n^{\circ}$ 26-27. pp. 123-148.

Bouvier, B. (2003). "Chinois et Français : quand les habitudes culturelles d'apprentissage s'opposent". ELA, 132, octobre-décembre 2003. pp. 399-414.

Clerc, S. (2002). "L'acquisition des conduites narratives en français langue étrangère". In Cicurel, F. \& Véronique, D. Discours, action et appropriation des langues. Paris : Presses Sorbonne Nouvelle.

Collie, J. \& Slater, S. (1987). Literature in the language classroom. Cambridge : Cambridge University Press.

Conseil de l'Europe. (2001). Cadre Européen Commun de Référence pour les langues : apprendre, enseigner, évaluer (CECR). Paris: Les Editions Didier.

Corson, D. J. (1985). The lexical Bar. Oxford: Pergamon Press.

Courtillon, J. (1989). » Lexique et apprentissage de la langue. » Le français dans le monde. Recherches et applications, août-septembre 1989. pp. 146-153.

Cuq, J. P. (2003). Dictionnaire de didactique du français langue étrangère et seconde, Paris : CLE International.

Cuq, J. P. et Gruca, I. (2005). Cours de didactique du français langue étrangère et seconde. Grenoble : Presses Universitaires de Grenoble.

Dufays, J. \& Plane, S. (2009). L'écriture de fiction en classe de français. Namur : Presses Universitaires de Namur. 
Elalouf, M. \& Kéravent, J. (2004). "L'acquisition du lexique à l'épreuve d'un grand corpus de textes d'élèves". In Calaque, E. \& David, J. Didactique du lexique, contextes, démarches, supports. Bruxelles : De Boeck.

Guillot, B. (2010). Les représentations sociales de l'écriture et le rapport à l'écriture en langue étrangère : une approche didactique pour l'enseignement et l'apprentissage de l'écrit en FLE en milieu universitaire australien. The Australian National University, Canberra. Thèse de doctorat non publiée.

Ionnidou, A. (2008)."La compétence lexicale dans le Cadre européen commun de référence pour les langues." Travaux de didactique du FLE, n 59, pp. 59-69.

Laufer, B. (1994) "Appropriation du vocabulaire : Mots faciles, mots difficiles, mots impossibles." Aile, $\mathrm{n}^{\circ}$ 3, pp. 97-113.

Lefrançois, P. (2001)."Le point sur les transferts en écriture en langue seconde". The Canadian Modern Language Review. La revue canadienne des langues vivantes, ${ }^{\circ}$ 58. pp. 223-245.

Odlin, T. (1989). Language transfer. Cross-linguistic influence in language learning. Cambridge: Cambridge University Press.

Nation, I. (2001). Learning Vocabulary in Another Language. Cambridge: Cambridge University Press.

Pandraud, N. (2009). "Apprendre à devenir auteur d'un récit de fiction. Observations de l'écriture

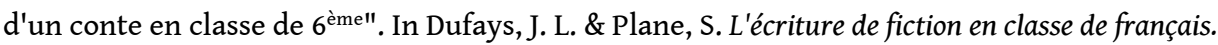
Namur : Presses Universitaires de Namur.

Pilorgé, J.-L. (2010). "Un lieu de tension entre posture de lecteur et posture de correcteur : les traces des enseignants de français sur les copies d'élève". Pratiques, n 145. pp. 85-103.

Pintado, C. (2006). Les Contes de Perrault à l'épreuve du détournement dans la littérature de jeunesse de 1970 à nos jours. De la production à la réception. Rennes : Université Rennes 2 Haute-Bretagne.

Sardier, A. \& Grossmann, F. (2010). "Comment favoriser le réemploi lexical ?" Recherches, $\mathrm{n}^{\circ}$ 53, 2010-12. pp. 9-34.

Tauveron, C. (1995). Le personnage. Une clef pour la didactique du récit à l'école élémentaire. Genève : Neuchâtel et Paris, Delachaux et Niestlé.

Treville, M-C. (1996). « Le niveau de perfectionnement : le cas du vocabulaire de la langue seconde ». In Courchêne et alii, Vingt ans d'enseignement des langues secondes à l'université d'Ottawa. pp. 92-110.

Treville, M-C. \& Duquette, L. (1996). Enseigner le vocabulaire en classe de langue. Paris : Hachette.

\section{Littérature pour la jeunesse}

Pommaux, Y. (1993). John Chatterton détective. Paris : L'école des loisirs.

Solotareff, G. \& Nadja. (1989). Le Petit Chaperon Vert. Paris : L'école des loisirs.

\section{Ouvrage de FLE}

Denisot, H. \& Piquet, M. (2002). Tatou le Matou. Paris : Hachette FLE. 


\section{NOTES}

1. "Pour situer le programme par rapport au Cadre Européen Commun de références pour les Langues (CECR), les étudiants du programme atteignent le niveau A2 au cours ou en fin de premier semestre de deuxième année. Le niveau B1 est atteint par les étudiants du cursus en milieu de troisième année. Le niveau B2 est atteint en fin de quatrième année. Dans certains cas, et généralement en fonction de leurs séjours en pays francophone ou de leur parcours avant leur intégration au programme, certains étudiants peuvent atteindre le niveau C1 en fin de cursus" (Guillot, 2010 : 79).

2. On pourra se référer ici au numéro 73, 2008 de Recherches et travaux sur l'écriture d'invention.

3. Au niveau A2 l'apprenant peut, selon le CECR, "écrire une série d'expressions ou de phrases simples reliées par des connecteurs simples tels que 'et', 'mais' et 'parce que'". Pour ce qui est de l'écriture créative, le CECR indique qu'au niveau A2 l'apprenant peut (i) écrire sur les aspects quotidiens de son environnement, par exemple les gens, les lieux, le travail ou les études avec des phrases reliées entre elles; (ii) faire une description brève et élémentaire d'un évènement, d'activités passées et d'expériences personnelles; (iii) écrire une suite de phrases et d'expressions simples sur sa famille, ses conditions de vie, sa formation, son travail actuel ou le dernier en date; (iv) écrire des biographies imaginaires et des poèmes courts et simples sur les gens (Conseil de l'Europe, $2001: 52$ ).

\section{RÉSUMÉS}

Le présent article interroge la pertinence des critères habituellement retenus pour l'évaluation de la compétence lexicale dans les productions écrites. Il se base sur une recherche doctorale conduite au premier semestre 2009 à l'Australian National University (ANU) (Canberra) et met en évidence l'insuffisance des critères de maîtrise et d'étendue proposés par le Cadre Européen Commun de Référence pour les langues (CECR) pour rendre compte de l'appropriation du lexique dans le cadre d'exercices de type écriture sous contraintes qui s'inscrivent dans le prolongement de lectures d'iconotextes adressés à la jeunesse. Nous proposons ici d'établir un critère supplémentaire qui permet de faire ressortir le travail linguistique opéré par l'apprenant scripteur en langue étrangère et de mettre en valeur l'apport personnel de celui-ci dans des productions monomodales caractérisées par une profonde hybridité.

This article examines the pertinence of the criteria that are usually applied to the evaluation of lexical competency in written language production. It is based on doctoral research undertaken during the first semester of 2009 at The Australian National University (ANU) (Canberra) and highlights the inadequacy of the two criteria of mastery and broadness of the lexical repertoire elaborated in the Common European Framework of Reference for Languages (CEFR) in assessing the extent to which vocabulary acquisition is evidenced through constrained writing exercises that follow on from the reading of iconotexts written originally for children. An additional criterion is proposed in order to emphasise the extent of the linguistic task performed by the student writing in a foreign language and to highlight the individual contribution made by the student in a monomodal piece of writing that displays considerable hybridity. 
INDEX

Thèmes : Varia

Keywords : vocabulary acquisition, CEFR, assessment, constrained writing, hybridity, complexity Mots-clés : appropriation du lexique, CECR, évaluation, écriture sous contraintes, hybridité, complexité

\section{AUTEUR}

\section{CHRISTÈLE MAIZONNIAUX}

Christèle Maizonniaux est enseignante-chercheuse à l'Université Flinders (Adélaïde, Australie). Sa thèse de doctorat, réalisée en cotutelle (ANU, Canberra et Université Stendhal-Grenoble 3), a été soutenue en 2013. Elle porte sur les apports de la littérature de jeunesse pour la didactique du FLE en contexte universitaire australien.

Courriel : christele.maizonniaux@flinders.edu.au Toile : http://www.flinders.edu.au/people/christele.maizonniaux Adresse : Flinders University. School of Humanities and Creative Arts. Department of Languages and Applied Linguistics. Room 2. 09. Adelaide, SA 5001 Australia. 